Samuel Höing ${ }^{*}$, Finja Borowski, Jan Oldenburg, Sabine Illner, Alper Öner, Michael Stiehm, Klaus-Peter Schmitz and Sebastian Kaule

\title{
Particle Image Velocimetry for in vitro characterization of Paravalvular Leakage of TAVR-sealing concepts
}

\begin{abstract}
Paravalvular leakage (PVL), defined as the leakage between the aortic annulus and a transcatheter aortic valve replacement (TAVR), is verifiably associated with short- and long-term clinical outcome, especially with increased mortality. Therefore, with the ambition to reduce or even prevent PVL of next generation TAVR, it is necessary to extend the hemodynamic understanding of PVL.

This study presents an in vitro flow measurement method to localize PVL during hydrodynamic characterization of TAVR and furthermore presents different design features, socalled outer skirt, to reduce PVL. Particle image velocimetry (PIV) measurements were performed for flow field assessment during hydrodynamic characterization of TAVR. Additionally, two different sealing concepts were developed to reduce PVL. The skirts were manufactured from polymeric-nonwoven and sued to pericardium-based TAVR-prototype. The prepared TAVR-prototypes were then deployed in a pathophysiological model of the aortic root with a calcification nodule of $2 \mathrm{~mm}$ according to ISO 5840:2021. To assess PVL, the flow field and the regurgitation volume was measured.

The PIV measurements showed a clearly visible leakage jet between the TAVR-prototypes without skirt and the pathophysiological aortic annulus model. Jet velocities of up to 0.5 $\mathrm{m} / \mathrm{s}$ were measured depending on presence or configuration of a PVL-preventing skirt. When implanted in the physiological annulus model without calcification nodule, PVL was hardly recognizable. The regurgitation volume of a TAVR-prototype
\end{abstract}

\footnotetext{
*Corresponding author: Samuel Höing: Student at the Institute for Biomedical Engineering; Institute for ImplantTechnology and Biomaterials e. V., Rostock-Warnemünde, Germany, e-mail: samuel.hoeing@uni-rostock.de

Finja Borowski, Jan Oldenburg, Michael Stiehm, Klaus-Peter Schmitz, Sebastian Kaule: Institute for Implant Technology and Biomaterials e. V., Rostock-Warnemünde, Germany Sabine IIIner: Institute for Biomedical Engineering, Rostock University Medical Center, Rostock-Warnemünde, Germany Alper Öner: Department of Cardiology Rostock University Medical Center, Rostock, Germany
}

without skirt at $51 / \mathrm{min}$ was $36.26 \pm 1.89 \mathrm{ml}(\mathrm{n}=10)$. The developed and manufactured polymeric-nonwoven skirts reduced PVL from $37.67 \pm 1.17 \mathrm{ml}$ to $18.36 \pm 1.8 \mathrm{ml}(\mathrm{n}=10$, TAVR-skirt-design1) and from $46.97 \pm 1.07 \mathrm{ml}$ to $17.85 \pm 1.29 \mathrm{ml}(\mathrm{n}=10$, TAVR-skirt-design 2$)$ at $51 / \mathrm{min}$. The localization of PVL during hydrodynamic characterization by means of PIV was successful. The sealing concepts developed in this work were very effective and led to a PVL-reduction of the tested TAVR prototypes of about $50 \%$ to $70 \%$.

Keywords: Transcatheter Aortic valve, hydrodynamic testing, particle image velocimetry, paravalvular leakage.

https://doi.org/10.1515/cdbme-2021-2170

\section{Introduction}

Gold standard for the treatment of severe aortic valve stenosis or insufficiency is surgical aortic valve replacement (SAVR). If the surgical risk is too high for the patient, minimally invasive implantation of a transcatheter aortic valve replacement (TAVR) is the treatment of choice. While the number of surgical interventions decreases, TAVR implantations have been increasing continuously since 2008 in Germany [1]. Furthermore, the benefits of TAVR have also been demonstrated in patients with a low or intermediate surgical risk in several studies $[2,3]$.

Nevertheless, acute complications such as paravalvular leakage (PVL) can occur, which is associated with increased mortality $[4,5]$. To address this problem, recent TAVR devices feature a sealing system. The so-called outer skirt consists of tissue patches, which are attached to the TAVR stent and leaflets. The skirt prevents PVL defined as backflow of blood occurring between the aortic annulus and the TAVR. Under in vitro conditions, the hemodynamic performance of TAVR according to ISO 5840 is currently assessed by flow measurements in cardiovascular circulation loops [6]. The efficacy is determined by the regurgitation volume, i.e. the sum of the 
closure volume and the transvalvular leakage and PVL. However, it is not possible to differentiate between transvalvular leakage, PVL as well as closing volume.

To overcome this limitation, we used particle image velocimetry (PIV) to investigate the effectiveness of different designs of outer skirt sealing concepts on TAVR preventing PVL.

\section{Materials and Methods}

\subsection{Sealing concepts}

A close-fitting and a wide skirt concept were developed for further skirt analysis based on an analysis of existing skirt concepts. Both concepts consist of an electrospun nonwoven made of a thermoplastic Polyurethane-Elastomer (TPU-PC) and are sutured to the stent and the pericardium of the TAVR prototype, respectively.

The first concept pursues the close-fitting sealing concept and consists of a rectangular patch of TPU-PC covering the inflow area of the TAVR. An increased sealing is to be achieved by two double folded skirt rings (see Figure 1).

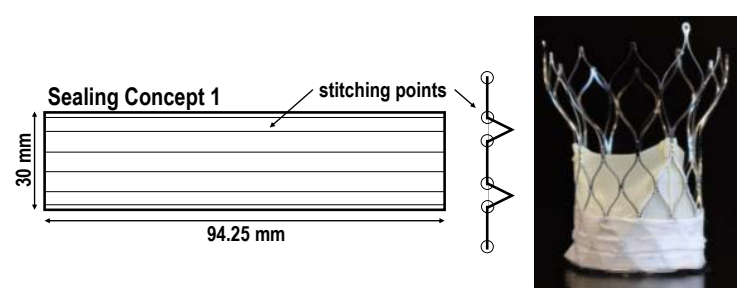

Figure 1: Contour of the skirt of the first sealing concept (left); sewn nonwoven on the TAVR prototype (right)

The second concept is based on the sealing of the SAPIEN 3 (Edwards Lifesciences Corporation, US). The pocket-structure result in a circular patch contour (see Figure 2).

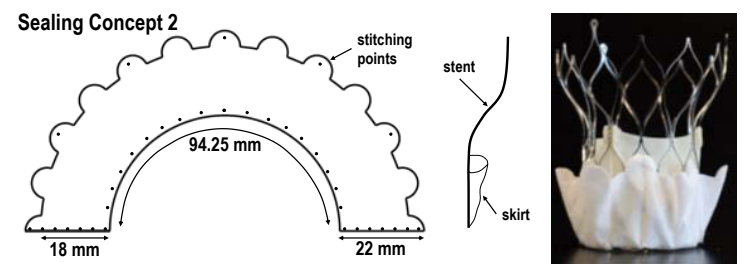

Figure 2: Contour of the skirt of the second sealing concept (left); sewn nonwoven on the TAVR prototype (right)

\subsection{Experimental setup}

To study the efficacy of the TAVR under in vitro conditions, a phantom model of the aortic root is needed. Based on the specifications of ISO 5840-3:2021, which provide guidance on the annulus and native leaflets, as well as previous work of our working group [7] a model was cast from silicone. The model takes into account the sinus valsalvae of the aortic root and has an inflow section proximal to the TAVR as well as an outflow section distal to the TAVR. The silicone is highly transparent, which ensures optical access. PVL was induced by introducing a calcification according to ISO5840-3:2021, with a nodule model of $2 \mathrm{~mm}$ width.

A commercially available cardiovascular circulation loop (Vivitro Labs, Victoria, CAN) was used in order to model a physiological pulsatile flow of the left heart. Since there is no optical access proximal to the TAVR in the standard configuration of the pulse duplicator system, a custom-made PIV acrylic test chamber was added to the system, which was adapted to the aortic root model. The test chamber opening allows a sizing of aortic root models that cover the anatomical conditions. All measurements were performed with the following hydrodynamic test parameters (see Table 1).

Table 1: Test parameters of the hydrodynamic and PIV measurements ( ${ }^{*}$ PIV measurements only at $5 \mathrm{l} / \mathrm{min}$ )

\section{Hydrodynamic parameter}

\begin{tabular}{lr}
\hline Temperature & $37 \pm 2{ }^{\circ} \mathrm{C}$ \\
Heart rate & $70 \mathrm{BPM}$ \\
Cardiac output & $3.0,5.0^{*}$ and $7.0 \mathrm{l} / \mathrm{min}$ \\
Mean aortic pressure & $100 \pm 2 \mathrm{mmHg}$ \\
Systolic duration & $35 \%$ \\
\hline
\end{tabular}

The regurgitation volumes of TAVR prototype with and without sealing were determined from the pressure and volume flow measurements with the pulse duplicator system.

For PIV measurements, the acrylic test chamber provides optical access for two PIV cameras, which are positioned by an angle of $45^{\circ}$ regarding to the laser light sheet for stereo PIV measurements (see Figure 3).

To enable distortion-free PIV measurements not only the optical accessibility, but also the optical distortion due to curved surfaces of the aortic root must be considered. For this purpose, the mixture of the blood substitute test fluid was adapted to the kinematic viscosity of $3.5 \mathrm{cSt}$ at $37^{\circ} \mathrm{C}$ as recommended by ISO $5840-3$ as well as by the refraction index of the used aortic silicone model. For this reason, a fluid mixture of $0.9 \%$ saline and glycerol with a mass fraction of glycerol of $50.6 \%$ was used.

A PIV system from DantecDynamics was used for the flow field evaluation. Two CMOS cameras (EoSens 12CXP+, Mikrotron $\mathrm{GmbH}, \mathrm{DE}$ ) with Scheimpflug adapter were used to capture the double frame images. The laser light sheet was induced by a double pulsed Nd:YAG dual-cavity laser (532 nm, max. energy 145 mJ, 15 Hz, Litron Laser Ltd., UK).

Fluorescent particles (micro particles $\mathrm{GmbH}, \mathrm{DE}$ ) were used to visualise the flow. With a size of $9.89 \mu \mathrm{m}$ and a density 
of $1.05 \mathrm{~kg} / \mathrm{m} 3$, non-slip flow tracking is assumed. Optical long-pass filters attached to the cameras capture only the emitted light of the particles

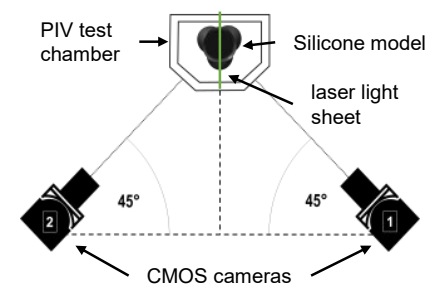

Figure 3: Experimental setup with two CMOS cameras at an angle of $45^{\circ}$ to the laser light sheet

The acquisition times of the PIV measurements were determined based on the pressure and volume flow measurements and high-speed recordings of the TAVR. Measurements were taken at a series of five time points within diastole at a cardiac output of $5 \mathrm{l} / \mathrm{min}$ and a mean aortic pressure of $100 \mathrm{mmHg}$.

The measuring area from the camera point of view is shown in red in Figure 4. To avoid random flow fluctuations, the acquisition was phase-averaged 100 times for each time point.

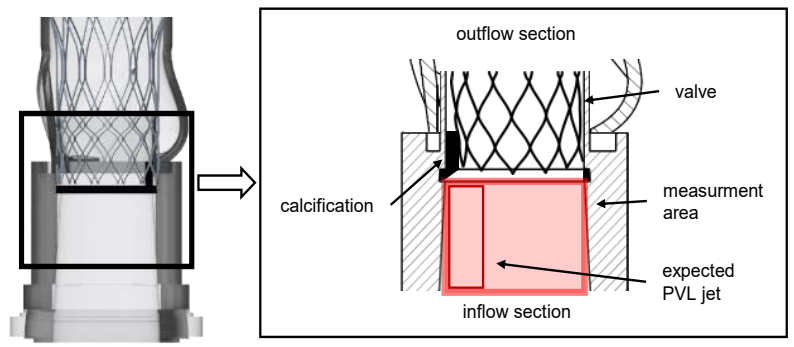

Figure 4: Illustration of the measuring area in the inflow section and the marked expected PVL jet area

\section{Results}

\subsection{Impact of sealing concepts on regurgitation volume}

Following, the sealing concepts are evaluated by the regurgitation volume under three different flow conditions - cardiac output volumes of 2.0, 5.0 and $7.0 \mathrm{l} / \mathrm{min}$.

Considering the ideal annulus TAVR without sealing concept were sufficient under the investigated cardiac output conditions. By deployment TAVR without sealing in pathological altered annulus a drastically increase in regurgitation volume was observed under all conditions. The TAVR device featuring sealing concept leads to a low regurgitation at a level of the ideal annulus. Both concepts showed similar efficacy (see Figure 5).
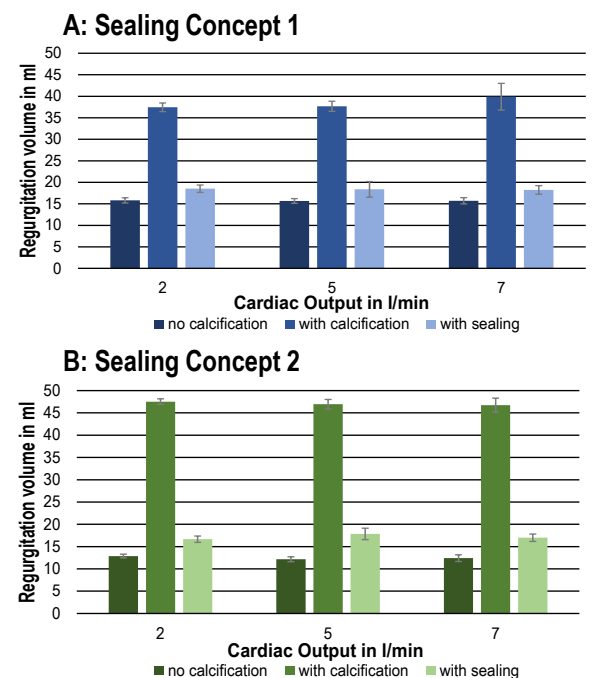

Figure 5: Regurgitation volumes of the prototypes determined in the pulse duplicator in the silicone model without and with calcification and in the silicone model with calcification and sealing concept 1 and 2

\subsection{Characterisation of the PVL with PIV}

Figure 6 depicts the velocity field proximal to the TAVR. Devices with and without sealing concepts were compared by extension and level of the leakage jet. Since the flow behaviour during diastole shows hardly any variation, two representative time points describing early-diastolic and late-diastolic were chosen.

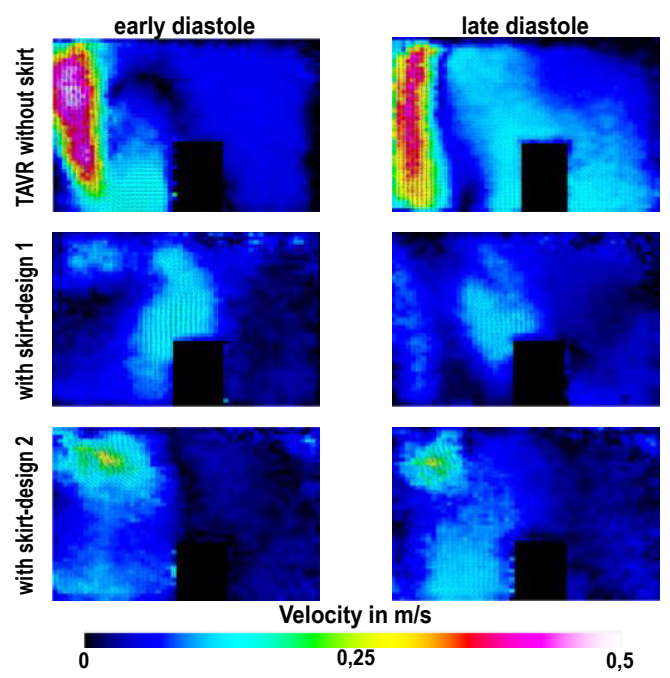

Figure 6: Comparison of the flow velocity fields of the PIV images of the TAVR prototype without sealing with the fields of sealing concepts 1 and 2 .

It can clearly be seen that TAVR without sealing is insufficient when deploying in the calcification annulus model. Jet velocities of $<0.5 \mathrm{~m} / \mathrm{s}$ were reached. The jet coil extends over the 
entire measurement area $(21.5 \mathrm{~mm})$. TAVR prototypes featuring sealing systems decrease the level of leakage by reduction of jet velocity (sealing 1: $>0.2 \mathrm{~m} / \mathrm{s}$ and sealing $2:>0.3 \mathrm{~m} / \mathrm{s}$ ) and size of the jet coil. The leakage occurs more diffusely and less as a centred jet. Despite the proven effectiveness of both sealing concepts, the flow velocity field of the second sealing concept shows higher velocities in the area of calcification.

\section{Discussion}

\subsection{Impact of the sealing concepts on the regurgitation volume}

Considering the regurgitation volume in the 5-stage classification system according to Pibarot et al. (2015) [8], calcification leads to an increased leakage of previously classified as mild to a moderate or rather moderate-to-severe leakage. Subsequently, the increased leakage could be reduced again to mildto-moderate by both sealing concepts. Moderate to severe PVL is common in clinical practice and is considered an unfavourable prognostic indicator for short- and long-term survival [9].

But, by using pulse duplicator measurements only the level of leakage and not the local distribution of the regurgitation flow can be observed. This aspect could, nevertheless, be useful for the development of even more effective sealing concepts.

\subsection{Characterization of the PVL with PIV}

The results of both measurement systems - pulse duplicator measurements and PVL - are in good agreement. As seen by pulse duplicator measurements, PIV also prove the efficacy of both sealing concepts regard to PVL, when TAVR prototypes were deployed in calcification annulus model.

PIV is suitable to capture velocity distribution of PVL and consequently, assumptions can be made as to whether the leakage is trans- or paravalvular. Furthermore, based on our studies an impact of severe PVL on systolic flow is hypothesized and should be investigated in further studies.

It has to be noted that 2D3C-PIV is performed for one measurement plan. PVL as 3D flow phenomena could not be captured in full complexity. Therefore, to evaluate design TAVR design and sealing concepts it is recommended to measure the velocity distribution in three imaging planes according to ISO 5840.

\section{Conclusion}

Sealing systems drastically reduce PVL of TAVR, in particular when deployed in pathological altered annulus. The measurement of the systemic regurgitation volume by means of the pulse duplicator is not sufficient for future TAVR design development. Due to the high relevance of PVL a more precise in vitro investigation of $\mathrm{PVL}$ is needed.

We could show that local flow field acquisition by PIV provide helpful information in terms of velocity distribution for leakage characterization, where other derived metrics, such as shear rate, could also be obtained.

Based on high resolution measurement of hemodynamic situation future design concepts of sealing can be derived. Therefore, we recommend PIV measurements in TAVR at a certain phase of the design developing process of future device generations.

\section{Author Statement}

Research funding: Financial support by the Federal Ministry of Education and Research within RESPONSE "Partnership for Innovation in Implant Technology" is gratefully acknowledged. Conflict of interest: Authors state no conflict of interest.

\section{References}

[1] Deutsche Herzstiftung e.V. (2020) Deutscher Herzbericht 2019. Sektorenübergreifende Versorgungsanalyse zur Kardiologie, Herzchirurgie und Kinderherzmedizin in Deutschland

[2] Mack MJ et al. (2019) Transcatheter Aortic-Valve Replacement with a Balloon-Expandable Valve in Low-Risk Patients; doi: 10.1056/NEJMoa1814052

[3] Søndergaard L et al (2016) Two-Year Outcomes in Patients with Severe Aortic Valve Stenosis Randomized to Transcatheter Versus Surgical Aortic Valve Replacement: The All-Comers Nordic Aortic Valve Intervention Randomized Clinical Trial. Circ Cardiovasc Interv 9(6); doi: 10.1161/CIRCINTERVENTIONS.115.003665

[4] Kodali SK et al. (2012) Two-year outcomes after transcatheter or surgical aortic-valve replacement; doi: 10.1056/NEJMoa1200384

[5] Pibarot $P$ et al. (2017) Association of Paravalvular Regurgitation With 1-Year Outcomes After Transcatheter Aortic Valve Replacement with the SAPIEN 3 Valve; doi: 10.1001/jamacardio.2017.3425

[6] Deutsches Institut für Normung ISO 5840-3: 2021, Cardiovascuar implants - Cardiac valve prostheses - Part 3: Heart valve substitutes implanted by transcatheter technique

[7] Borowski F et al. (2020) Investigations of flow alteration of commissural misalignment of TAVR using Particle Image Velocimetry; doi: 10.1515/cdbme-2020-3041

[8] Pibarot $P$ et al. (2015) Assessment of paravalvular regurgitation following TAVR: a proposal of unifying grading scheme; doi: 10.1016/j.jcmg.2015.01.008

[9] Athappan G et al. (2013) Incidence, Predictors, and Outcomes of Aortic Regurgitation After Transcatheter Aortic Valve Replacement; doi: 10.1016/j.jacc.2013.01.047 